\title{
MARINER 5 MEASUREMENTS OF ULTRAVIOLET EMISSION FROM THE GALAXY
}

\author{
CHARLES A. BARTH \\ Dept of Astro-Geophysics, and Laboratory for Atmospheric and Space Physics, \\ University of Colorado, Boulder, Colo., U.S.A.
}

\begin{abstract}
The Mariner 5 ultraviolet measurements obtained while the spacecraft was in interplanetary flight are interpreted as Lyman- $\alpha$ radiation. This radiation may arise from (1) the scattering of solar Lyman- $\alpha$ radiation by interplanetary atomic hydrogen, (2) the scattering of solar Lyman- $\alpha$ by atomic hydrogen that is present at the edge of the solar system, or (3) diffusely scattered Lyman- $\alpha$ radiation from the Galaxy. The Mariner 5 measurements show a symmetry with respect to the galactic equator which suggests that the major source of the observed emission is the diffuse galactic radiation. In the Münch model for the diffuse Lyman- $\alpha$ radiation from an Hi region, the sources of the Lyman- $\chi$ photons are stellar chromospheres, the photons are scattered a large number of times by interstellar atomic hydrogen, and are absorbed by interstellar dust. Some of the Mariner 5 measurements may be attributed to Lyman- $\alpha$ emission from an $\mathrm{H}_{\text {II }}$ region since the field of observation scanned the edge of the Gum nebula.
\end{abstract}

\section{Introduction}

Mariner 5, a planetary spacecraft, performed a number of ultraviolet observations while in interplanetary flight to Venus and beyond. In the several weeks following the encounter with Venus on October 19, 1967, and before the cessation of radio communications with the Earth on December 1, 1967, the spacecraft executed a series of manoeuvres designed to measure Lyman- $\alpha$ radiation emanating from the Galaxy. The ultraviolet photometer experiment which was designed to measure the distribution of atomic hydrogen in the outer atmosphere of Venus was used in these experiments (Barth et al., 1967). The observations were unique in that they were conducted in interplanetary space far from the hydrogen coronas of Venus and the Earth, and from a spacecraft that was rolling in such a way that the photometer field of view made a nearly perpendicular crossing of the galactic plane.

\section{Experimental}

The Mariner 5 ultraviolet photometer consisted of three separate photomultiplier tubes each with a caesium iodide photocathode and a lithium fluoride window. Two of the channels had their short wavelength responses limited by calcium fluoride and barium fluoride filters to provide wavelength bands of 1250-2200 $\AA$ and 1350$2200 \AA$, respectively. The channel without a filter responded in the 1050-2200 wavelength interval. The experimental technique, which was designed for planetary observations, used the difference in signals between the lithium fluoride and calcium fluoride channels as a measure of hydrogen Lyman- $\alpha 1216 \AA$ radiation. The difference in signals between the calcium fluoride and barium fluoride channels was a measure of the atomic oxygen $1304 \AA$ line. In astronomical observations all three 
channels respond to radiation from early type stars. It is, however, still possible to interpret the difference in signals between the lithium fluoride and calcium fluoride channels as Lyman- $\alpha$ radiation. All three channels had field of view limiters but no optics. The full field of view for the lithium fluoride channel as it responded to a point source was $6^{\circ}$ and for the other two channels was $2.4^{\circ}$.

The photometer was mounted on the spacecraft so that its view direction made an angle of $90^{\circ}$ with the spacecraft-Sun line and an angle of $95^{\circ}$ with the plane containing the Sun, the spacecraft, and the star Canopus. Since the spacecraft was usually stabilized on the Sun and Canopus, the ultraviolet photometer viewed a region near the ecliptic which slowly changed in ecliptic longitude as the spacecraft traversed its orbit about the Sun. On several occasions the spacecraft was released from stabilization on Canopus and rolled about the spacecraft-Sun line. During these manoeuvres, the ultraviolet photometer field of view swept along lines of ecliptic longitude. Two such series of rolls, one on November 7, 1967, and the other on November 19, 1967, are the basis of the observations reported in this paper. At these times the spacecraft was approximately $1 \times 10^{8} \mathrm{~km}$ from the Sun and over $5 \times 10^{6} \mathrm{~km}$ from Venus.

\section{Observations}

The regions of the celestial sphere that were observed during the rolling manoeuvres are shown in Figure 1, which is a chart of ultraviolet stars plotted in galactic coordinates. The relative size of the dots represents the magnitudes of the stars as they would be measured in the 1350-1600 $\AA$ wavelength region where the three ultraviolet photometer channels had their maximum response. The track of the November 7 roll began in the Southern galactic hemisphere and moved initially to decreasing longitude

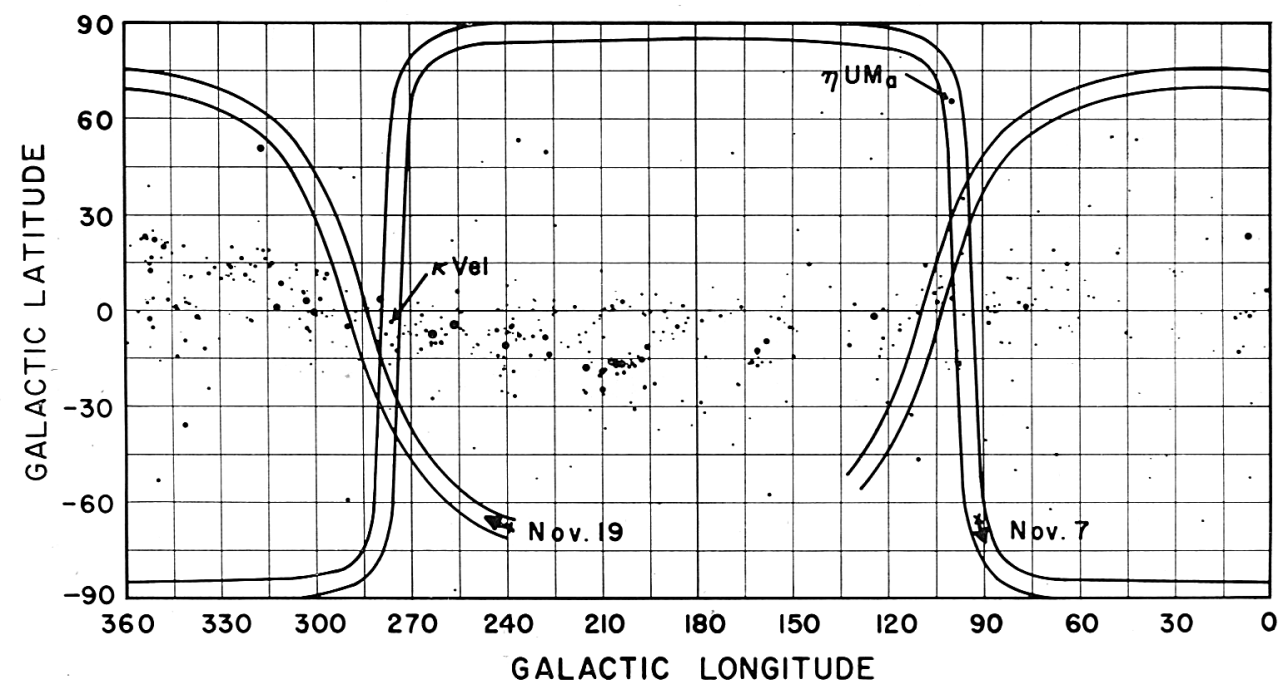

Fig. 1. Ultraviolet star chart which shows regions of observation during November 7 and 19 rolls. 
and then northward in latitude. The observation track crossed the plane of the Galaxy in the constellation Vela, sweeping to Northern galactic latitudes and then southward crossing the galactic plane again, this time in the constellation Cygnus. The width of track shown in the figure is $6^{\circ}$ corresponding to the full field of view of the lithium fluoride channel.

Data obtained on the November 7 roll are shown in Figure 2. The intensity of radiation measured by the lithium fluoride channel is shown as a linear function of the position in the roll. In addition, galactic latitude and ecliptic latitude coordinates are plotted along the abscissa. Figure 2 displays the data from three sequential rolls of the spacecraft, each approximately of 1 hour's duration. This lithium fluoride channel data shows responses to discrete ultraviolet objects such as the Large Magellanic Cloud, $\kappa$ Vel, and $\eta \mathrm{UMa}$. When the lithium fluoride channel responds to these objects, the calcium fluoride and barium fluoride channels respond as well. However, the lithium fluoride channel measures a signal throughout the roll which the other two do not. This signal is interpreted as Lyman- $\alpha$ radiation. The striking thing about this Lyman- $\alpha$ signal is that it is not uniform in all directions. To the South of the galactic plane, the flux is $5 \times 10^{-4}$ ergs $\mathrm{cm}^{-2} \mathrm{~s}^{-1} \mathrm{ster}^{-1}$, while to the North it is $7 \times 10^{-4} \mathrm{ergs} \mathrm{cm}^{-2} \mathrm{~s}^{-1} \mathrm{ster}^{-1}$. When the field of view crossed the galactic plane in Vela, an enhancement of Lyman- $\alpha$ flux was observed over a region $20^{\circ}$ wide. When the

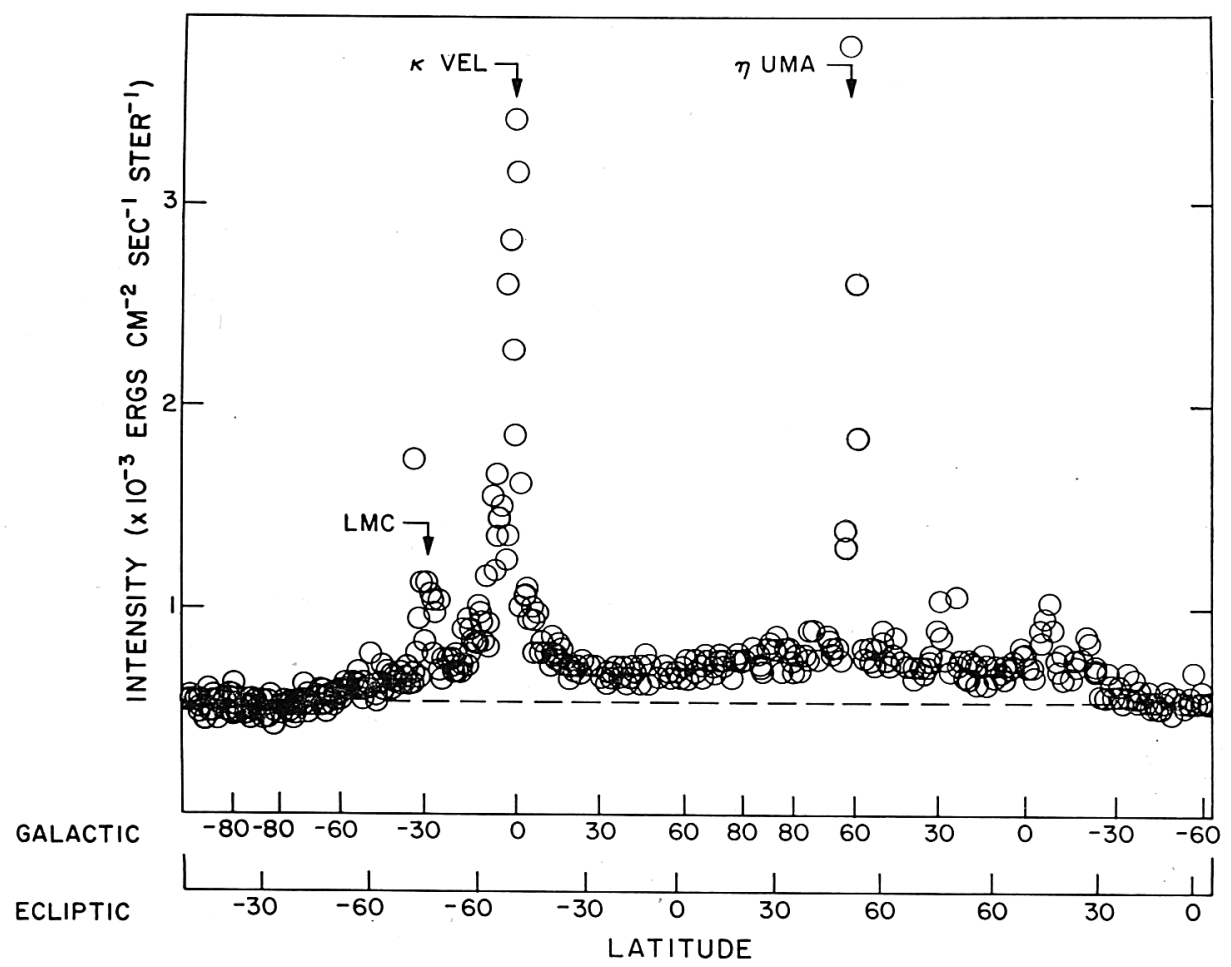

Fig. 2. Radiation measured by lithium fluoride channel during November 7 roll. 


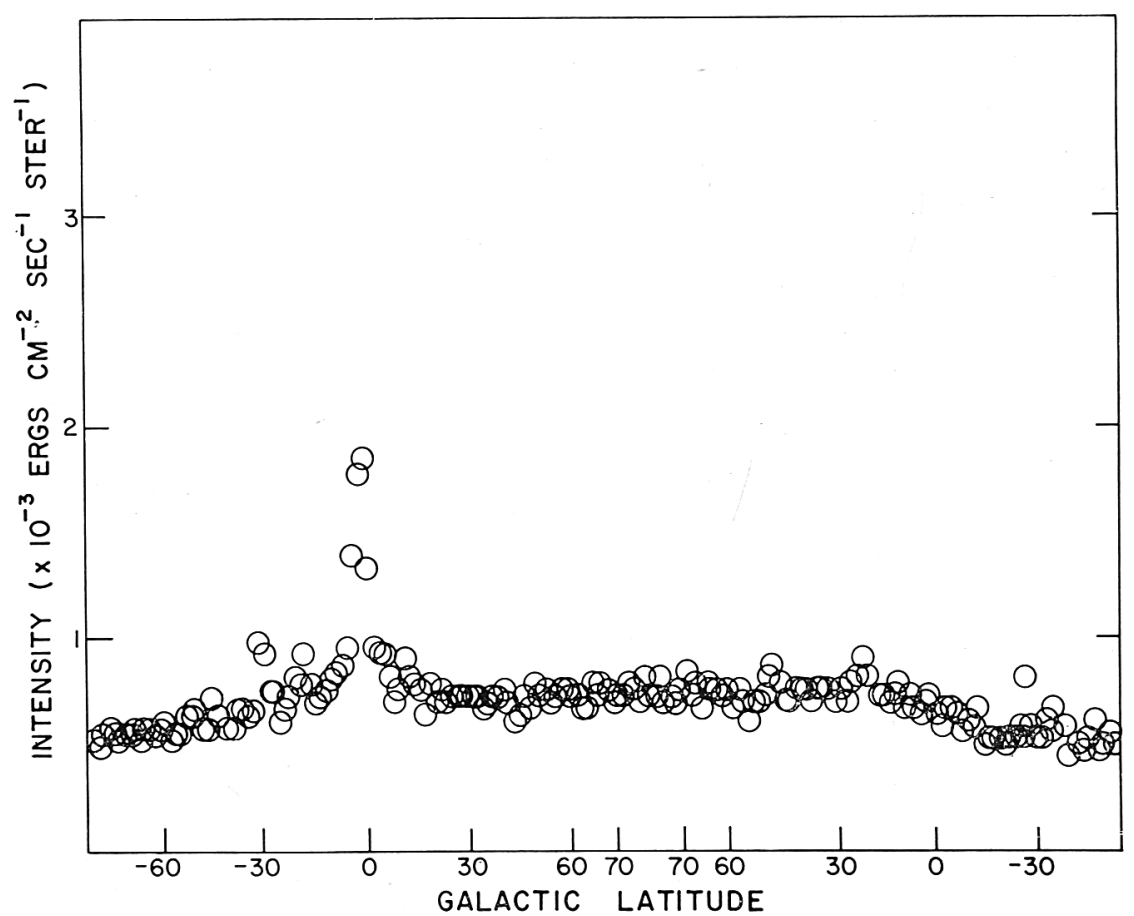

Fig. 3. Radiation measured by lithium fluoride channel during November 19 roll.

field of view crossed the galactic plane in Cygnus, however, a comparable enhancement was not observed.

The observation track of the November 19, 1967, roll is also shown in Figure 1. The observations also began in the Southern galactic hemisphere, crossed the galactic plane in Vela, scanned through the Northern galactic hemisphere crossing the plane again in Cygnus, stopping in the Southern hemisphere. This was a single roll of approximately $315^{\circ}$. The data from the lithium fluoride channel for this roll are shown in Figure 3. All of the general characteristics of the November 7 rolls are present. All three channels respond to hot stars and the Large Magellanic Cloud. The lithium fluoride channel alone shows a background signal throughout the roll. The Northern galactic hemisphere shows a larger value than the Southern hemisphere. The galactic plane crossing in Vela shows an enhancement in Lyman- $\alpha$, while the crossing in Cygnus does not.

\section{Interpretation}

The diffuse Lyman- $\alpha$ glow observed by Mariner 5 may originate from atomic hydrogen located (1) within the solar system, (2) at the edge of the solar system, or (3) in the interstellar medium. If resonance reradiation of solar Lyman- $\alpha$ by interplanetary atomic hydrogen is the explanation of the Mariner results, a density of the order of $10^{-2}$ atoms $\mathrm{cm}^{-3}$ is required if the atomic hydrogen is distributed uniformly through- 
out the solar system (Barth et al., 1968). For observations made perpendicular to the spacecraft-Sun line, as was the case with Mariner 5, the emission rate is equal to that produced by the local volume density of atoms times an equivalent path length of $\pi / 2$ times the distance of the instrument from the Sun. An interplanetary density of $1 \times 10^{-2}$ atoms $\mathrm{cm}^{-3}$ would produce an optical depth of approximately $\tau=0.1$, a value that is not in conflict with any spectroscopic observational results.

Even if the interplanetary medium is devoid of atomic hydrogen, there may be a buildup of atoms at the edge of the solar system where the solar wind runs into the interstellar medium (Patterson et al., 1963). For a shell of hydrogen atoms at $10 \mathrm{AU}$ illuminated by solar Lyman- $\alpha$, a density of $10^{-1}$ atoms $\mathrm{cm}^{-3}$ is required to explain the observed Mariner 5 intensities.

These two mechanisms in which the hydrogen atoms scatter Lyman- $\alpha$ radiation which originates in the Sun predict that the diffuse Lyman- $\alpha$ glow be distributed uniformly as viewed from the Mariner spacecraft or at most with a symmetry that is oriented with respect to an ecliptic coordinate system. The observations that are plotted in Figure 2 show that the asymmetric intensity distribution is oriented with respect to the galactic coordinate system and not the ecliptic. This observational result suggests that at least some, if not all, of the Lyman- $\alpha$ radiation must come from the Galaxy.

A model of diffuse galactic Lyman- $\alpha$ radiation has been given by Münch (1962). In this model, the source of the Lyman- $\alpha$ photons is the chromospheres of late type stars. The stellar Lyman- $\alpha$ photons are scattered by interstellar atomic hydrogen until they are finally absorbed by interstellar dust. Münch (1962) has calculated that the mean distance that a Lyman- $\alpha$ photon travels before being absorbed is $50 \mathrm{pc}$. In traversing that distance some $10^{9}$ scatterings occur. Münch also calculated that the expected surface brightness of the diffuse Lyman- $\alpha$ radiation should be $6.4 \times 10^{-4} \mathrm{erg} \mathrm{cm}^{-2}$ $\mathrm{s}^{-1}$ ster $^{-1}$. That this number agrees so well with the Mariner observations is surprising but perhaps fortuitous.

To explain the different intensities observed by Mariner 5 in the Northern and Southern galactic hemispheres using the Münch model, one may assume that the ratio of interstellar hydrogen to dust is different above the galactic plane than it is below the plane.

Münch (1962) has also pointed out that large local sources of Lyman- $\alpha$ radiation may be expected from $\mathrm{HII}$ regions, in particular, the extensive Gum HII region that is excited by $\gamma$ Vel and $\zeta$ Pup (Gum, 1952). In an Hil region, the ultimate source of photons is stellar flux shortward of the Lyman limit from early type stars. Lyman- $\alpha$ photons are produced during the radiative recombination of ionized hydrogen. The Lyman- $\alpha$ photons are lost in the $\mathrm{H}$ II region either by two-photon decay or ionization of the excited atom. Lyman- $\alpha$ photons escaping from the Hir region eventually are lost by absorption on interstellar dust.

The increase in the diffuse Lyman- $\alpha$ glow that occurred when the Mariner 5 photometer swept through Vela may be an observation of the edge of the Gum Hil region. Unfortunately, it was not possible to obtain observations closer to the center of this 
region from Mariner 5. If the identification of the brightening of the Lyman- $\alpha$ glow with the Gum region can be verified by future observations, it may be possible to determine how close this $\mathrm{H}$ II region extends to the solar system.

Observations of Lyman- $\alpha$ radiation were also made in interplanetary space from Venera 4 (Kurt and Dostovalov, 1968). The intensities observed from this spacecraft were about a factor of 3 lower than those observed from Mariner 5. It has not been possible to compare results from the two spacecraft as a function of galactic coordinates.

\section{Summary}

The diffuse Lyman- $\alpha$ glow observed by Mariner 5 is attributed at least in part to radiation scattered by hydrogen atoms in the interstellar medium. In addition, Lyman- $\alpha$ radiation from an $\mathrm{H}_{\text {II }}$ region may have been observed in Vela.

\section{Acknowledgement}

The Mariner 5 project is to be commended for conducting the operations necessary for these astrophysical observations after the completion of the planetary mission. This research has been supported by the National Aeronautics and Space Administration under grant number NGL 06-003-052.

\section{References}

Barth, C. A., Pearce, J. B., Kelly, K. K., Wallace, L., and Fastie, W. G. 1967, Science 158, 1675.

Barth, C. A., Wallace, L., and Pearce, J. B.: 1968, J. Geophys. Res. 73, 2541.

Gum, C.S.: 1952, Observatory 72, 151.

Kurt, V. G. and Dostovalov, S. B.: 1968, Nature 218, 258.

Münch, G.: 1962, in Space Age Astronomy (ed. by A. J. Deutsch and W. B. Klemperer), Academic Press, New York, ch. 10, p. 219.

Patterson, T. N. L., Johnson, F. S., and Hanson, W. B.: 1963, Planet. Space Sci. 11, 767.

\section{Discussion}

Severny: I have two questions. First: How do you explain the high increase in Lyman- $\alpha$ intensity in high galactic latitudes? Second: do you observe the increase of intensity outside Lyman- $\alpha$ when crossing the Milky Way?

Barth: If the model put forth by Münch (1962) is the correct explanation of the galactic Lyman- $\alpha$ intensity, then the difference in intensity between the Northern and Southern galactic hemispheres may be explained by a difference in the ratio of neutral hydrogen to dust in these two regions. The photometer channels that measured outside of the Lyman- $\alpha$ region did not measure an increase in signal when crossing the Milky Way other than that produced by early stars.

Carruthers: What density of interstellar hydrogen was assumed in deriving the $50 \mathrm{pc}$ mean free path for Lyman- $\alpha$ scattering?

Barth: In the calculations that were done by Münch (1962) for Lyman- $\alpha$ scattering in an H I region, the density of interstellar hydrogen that was used was 1 atom $\mathrm{cm}^{-3}$.

Carruthers: If the density is $0.1 \mathrm{~cm}^{-3}$ instead of $1.0 \mathrm{~cm}^{-3}$, then the scattering path would be comparable with the thickness of the galactic disk, and hence one would more likely expect an increase in the observed emission toward the galactic plane.

Underhill: What constellation did you cross through when going from the $\mathrm{N}$ side of the galactic plane to the $\mathrm{S}$ side? 
Barth: Cygnus.

Henize: Can you give any estimate of the densities required if the Vela radiation originates in the Gum Hil region?

Barth: There does not seem to be much doubt that $\mathrm{H}$ II regions will produce Lyman- $\alpha$ radiation. The key question in the case of the Gum H H region is whether there is an intervening Hi region of sufficient density to obscure the Lyman- $\alpha$. This problem can be solved if additional observations are made in the Vela-Puppis region.

Henize: I note a large deflection due to the Large Magellanic Cloud. Is it possible to use this data to estimate the ability of the Lyman- $\alpha$ radiation to penetrate the interstellar medium?

Barth: The observation of galaxies in Lyman- $\alpha$ radiation is potentially a powerful technique to learn something about the density of intergalactic hydrogen. A preliminary examination of the data in all three channels from the Mariner 5 photometer has not revealed any excess Lyman- $\alpha$. That may be attributed to the Large Magellanic Cloud. The signal that you see in the figure may be explained as coming from a large number of early type stars.

Herbig: If the mean free path of a Lyman- $\alpha$ photon is so short in the interstellar medium, would it not be more significant to correlate these data not with galactic latitude, but with latitude measured with respect to the plane of Gould's Belt?

Barth: That is an excellent suggestion.

Sunayev: (a) The stars of the Galaxy must give emission both in the Lyman- $\alpha$ line and in the continuous spectrum. In this case the response of 'Venus' counters would be commensurate, whereas their ratio $[(1050-1340 \AA) /(1222-1340 \AA)]$ is 100 .

(b) Gaseous nebulae and the aggregate of $\mathrm{H}$ II regions surrounding hot stars also cannot ensure the observed intensity of Lyman- $\alpha$ emission since their emission is the result of the redistribution of stellar radiation beyond the Lyman limit, which cannot significantly (100-1000 times) exceed the total emission in the 1225-1340 $\AA$ band (Kurt's measurements).

(c) Subcosmic ray ionization losses in the interstellar medium are not sufficient because their energy density is not high enough.

Probably all Lyman- $\alpha$ radiation originates from the interaction of the stellar wind due to a mass loss with the interstellar medium. In this case the width of the line is very large and shifted Lyman- $\alpha$ quanta reached the observer without scattering in $\mathrm{HI}$ regions. The other possibility is that the origin of this Lyman- $\alpha$ radiation is in the inter planetary medium. It may be that the density of the solar wind is higher in the galactic plane because of the influence of the Galactic magnetic field on the solar wind. 